Original Article

\title{
Melatonin modulates nitric oxide-regulated WNK-SPAK/OSR1- NKCC1 signaling in dorsal raphe nucleus of rats
}

\author{
Hye Jin Yang ${ }^{1, \#}$, Mi Jung Kim ${ }^{2,3, \#}$, Sung Soo Kim ${ }^{3,4}$, and Young-Wuk Cho ${ }^{1,2,3, *}$ \\ 'Department of Biomedical Science, Graduate School, Kyung Hee University, ${ }^{2}$ Department of Physiology, College of Medicine, Kyung Hee University, \\ ${ }^{3}$ Biomedical Science Institute and Medical Research Center for Reactive Oxygen Species, College of Medicine, Kyung Hee University, ${ }^{4}$ Department of Biochemistry \\ and Molecular Biology, College of Medicine, Kyung Hee University, Seoul 02447, Korea
}

\author{
ARTICLE INFO \\ Received March 11, 2021 \\ Revised June 21, 2021 \\ Accepted July 20, 2021 \\ *Correspondence \\ Young-Wuk Cho \\ E-mail:ywcho@khu.ac.kr
}

\section{Key Words}

Dorsal raphe nucleus

Melatonin

Nitric oxide

Sodium potassium chloride cotransporter WNK

\#These authors contributed equally to this work.

\begin{abstract}
The sleep-wake cycle is regulated by the alternating activity of sleep- and wake-promoting neurons. The dorsal raphe nucleus (DRN) secretes 5-hydroxytryptamine (5-HT, serotonin), promoting wakefulness. Melatonin secreted from the pineal gland also promotes wakefulness in rats. Our laboratory recently demonstrated that daily changes in nitric oxide (NO) production regulates a signaling pathway involving with-no-lysine kinase (WNK), Ste20-related proline alanine rich kinase (SPAK)/oxidative stress response kinase 1 (OSR1), and cation-chloride co-transporters (CCC) in rat DRN serotonergic neurons. This study was designed to investigate the effect of melatonin on NO-regulated WNK-SPAK/OSR1-CCC signaling in wake-inducing DRN neurons to elucidate the mechanism underlying melatonin's wake-promoting actions in rats. Ex vivo treatment of DRN slices with melatonin suppressed neuronal nitric oxide synthase (nNOS) expression and increased WNK4 expression without altering WNK1, 2, or 3. Melatonin increased phosphorylation of OSR1 and the expression of sodium-potassium-chloride co-transporter 1 (NKCC1), while potassium-chloride cotransporter 2 (KCC2) remained unchanged. Melatonin increased the expression of tryptophan hydroxylase 2 ( $\mathrm{TPH} 2$, serotonin-synthesizing enzyme). The present study suggests that melatonin may promote its wakefulness by modulating NO-regulated WNK-SPAK/OSR1-KNCC1 signaling in rat DRN serotonergic neurons.
\end{abstract}

\section{INTRODUCTION}

Regulation of the sleep-wake cycle is important for the maintenance of physical, mental, and emotional health [1,2]. The restorative role of sleep is responsible for recuperation from oxidative stress or toxic effects that have accumulated during wakefulness [3]. The homeostatic process of sleep-wake cycle regulation is controlled by alternating the activity of sleep- and wake-promoting neurons and by various neuronal networks and neurotransmitters in the brain [4]. Ventrolateral preoptic area (VLPO) neurons of the hypothalamus release gamma-aminobutyric acid (GABA) and act as sleep-inducing neurons, whereas neurons of the dorsal raphe nucleus (DRN) reportedly promote wakefulness [5-7].
DRN neurons are representative wake-active neurons containing the highest density of 5-hydroxytryptamine (5-HT, serotonin) cell bodies in the brainstem raphe nuclei [8]. 5-HT induces wakefulness and inhibits rapid eye movement (REM) sleep [9]. The firing rate of DRN serotonergic neurons remains consistent during wakefulness, decreases during slow wave sleep (SWS), and practically ceases during REM sleep [7,10]. SWS suppression of DRN neuronal firing originates in VLPO postsynaptic inhibition through 5-HT receptors [5,7].

DRN neuronal activity is modulated by GABAergic synaptic inputs [11]. The inhibitory effect of GABAergic synaptic inputs is altered by intracellular $\mathrm{Cl}^{-}$concentration that is regulated by cation-chloride co-transporters (CCC), including $\mathrm{K}^{+}-\mathrm{Cl}^{-}$cotrans- (i) \$ This is an Open Access article distributed under the terms of the Creative Commons Attribution Non-Commercial License, which permits unrestricted non-commercial use, distribution, and reproduction in any medium, provided the original work is properly cited. Copyright @ Korean J Physiol Pharmacol, pISSN 1226-4512, elSSN 2093-3827
Author contributions: H.J.Y. and M.J.K. equally contributed to this work and performed the experiments. H.J.Y. and Y.W.C. wrote the manuscript. Y.W.C. and S.S.K. designed and supervised the study. 
porter 2 (KCC2) and $\mathrm{Na}^{+}-\mathrm{K}^{+}-\mathrm{Cl}^{-}$cotransporter 1 (NKCC1) [12-14]. With-no-lysine kinases (WNKs) regulate the activity of KCC and NKCC by phosphorylating them directly or through Ste20-related proline alanine rich kinase (SPAK)/oxidative stress response kinase 1 (OSR1) signaling pathways [15-19].

Nitric oxide (NO) has a well-established role in the regulation of the sleep-wake cycle $[7,20]$. Neuronal nitric oxide synthase (nNOS) and inducible nitric oxide synthase (iNOS) regulate REM sleep in an opposing manner [21]. It has been shown that upregulation of iNOS associated with the immune response induces nonrapid eye movement (NREM) sleep during infections [22]. Neuromodulators such as NO, adenosine, growth factors, and cytokines have been suggested as endogenous sleep-inducing substances [7]. SWS and REM sleep were induced by treatment with NO donors [23]. A recent study from our laboratory demonstrated that daily changes in NO production regulate WNK-SPAK/OSR1CCC signaling in DRN serotonergic neurons [24]. However, the mechanism regulating daily changes in NO production in DRN neurons during the sleep-wake cycle remain unelucidated.

Melatonin is a neurohormone that regulates the sleep-wake cycle [25]. Melatonin is synthesized from serotonin and is secreted by the pineal gland [26], a process which is controlled by the circadian clock in the suprachiasmatic nucleus (SCN) of the hypothalamus and is directly inhibited by light [27,28]. Melatonin release peaks at midnight and dawn, and falls during the day [26]. While high levels of melatonin released during the night induces sleep in humans [29], melatonin reduces REM and SWS sleep and increased wakefulness in rats [30]. The mechanisms underlying melatonin's action is reportedly closely related to the circadian sleep regulation of SCN: the pineal gland and SCN interact have been shown to interact [31,32], and melatonin receptors are expressed in the SCN $[33,34]$. However, the effect of melatonin on homeostatic sleep regulation controlled by sleep- or wakeinducing neurons remains unclear. The present study therefore investigated the hypothesis that melatonin promotes wakefulness through the action of wake-inducing DRN serotonergic neurons and that melatonin functions as an initiating regulator of NOregulated WNK-SPAK/OSR1-CCC signaling in DRN neurons of the rat.

\section{METHODS}

\section{Animal handling}

Sprague-Dawley rats (either sex, 20-21 days-old) from Orient Bio (Seongnam, Korea) were used for experimentation. Rats were housed in a temperature-controlled vivarium $\left(22^{\circ} \mathrm{C}-24^{\circ} \mathrm{C}\right)$ with free access to food and water. All experiments were approved by the Kyung Hee University Animal Research Polices Committee (approval No. KHUASP(SE)-16-054) and conformed to the guidelines of the Council of the Korean Physiology Society and the National Institutes of Health Guide for the Care and Use of Laboratory Animals. Animals were adapted to a $12 \mathrm{~h}$ light-dark cycle (lights on at 8:00 am, lights off at 8:00 pm) at least a week before the commencement of experiments.

\section{Preparation of brain slices and tissue collection}

Brain slices including the DRN were prepared as described previously $[24,35]$. Briefly, animals were anesthetized with urethane (1.2 g/kg, i.p.; Sigma-Aldrich, St. Louis, MO, USA) [36]. Brains were quickly removed from the skull and immersed into an icecold artificial cerebrospinal fluid (aCSF) containing $124 \mathrm{mM}$ $\mathrm{NaCl}, 1.3 \mathrm{mM} \mathrm{MgSO}_{4}, 3 \mathrm{mM} \mathrm{KCl}, 1.25 \mathrm{mM} \mathrm{NaH}_{2} \mathrm{PO}_{4}, 26 \mathrm{mM}$ $\mathrm{NaHCO}_{3}, 2.4 \mathrm{mM} \mathrm{CaCl}_{2}$, and $10 \mathrm{mM}$ glucose (bubbled with $95 \%$ $\mathrm{O}_{2}$ and $5 \% \mathrm{CO}_{2}$ ). Whole brains were sliced coronally with a vibratome (VT1200, Leica Microsystems; Nussloch GmbH, Nussloch, Germany), and 400- $\mu \mathrm{m}$ thick slices containing the DRN were prepared.

\section{Melatonin treatment}

DRN slices $(400-\mu \mathrm{m})$ were immersed in an ice-cold aCSF solution (bubbled with 95\% $\mathrm{O}_{2}$ and 5\% $\mathrm{CO}_{2}$ ) containing 0.1, 1, and $10 \mu \mathrm{M}$ of melatonin (Sigma-Aldrich Korea, Seoul, Korea) for 4 h. After the incubation, DRN-containing tissues were collected for western blotting. Based on our previous report, melatonin treatment was started at 12:00 pm when the expression of nNOS began to increase [24].

\section{Western blotting}

The DRN region was punched out from slices under a binocular microscope (SZ-ST; Olympus, Tokyo, Japan) according to the rat brain atlas [37]. Because the size of the DRN region is small, DRN tissues from four rats should be pooled to form a single sample. A single group (N) consisted of $0.1,1$, and $10 \mu \mathrm{M}$ of melatonin-treated samples, including a control. Western blotting was performed according to our previous report [38]. Briefly, each DRN sample was lysed with proprep (iNtRON Biotechnology, Seongnam, Korea) in ice-cold conditions $\left(4^{\circ} \mathrm{C}\right)$ for $20 \mathrm{~min}$. The protein concentration of each sample was determined with Bradford assay. Samples (30 $\mu$ g of protein) were resolved on an $8 \%$ SDS-polyacrylamide gel, transferred to a nitrocellulose membrane, and blocked for $1 \mathrm{~h}$ in $5 \%$ skimmed milk at room temperature $\left(20^{\circ} \mathrm{C}-25^{\circ} \mathrm{C}\right)$. After $1 \mathrm{~h}$ of blocking, the membrane was reacted sequentially with primary antibody (anti-WNK1, anti-WNK2, anti-WNK3, anti-WNK4, anti-SPAK, anti-OSR1, anti-phosphoSPAK/OSR1, anti-NKCC1, anti-KCC2, anti-nNOS, anti-TPH2, or anti- $\beta$-actin) for overnight incubation at $4^{\circ} \mathrm{C}$. After washing with tris-buffered saline with Tween 20 (TBS-T) containing $25 \mathrm{mM}$ Tris-Cl, $150 \mathrm{mM} \mathrm{NaCl}$, and 0.05\% Tween-20, blots were incubated for $2 \mathrm{~h}$ in HRP-conjugated secondary antibodies (anti-rabbit or 
anti-mouse IgG). Immunoreactivity was visualized with a Western Blotting Detection Reagent Kit (Abclon, Seoul, Korea). Antibody binding was determined with chemiluminescent detection system (Davinch-Chemi, Seoul, Korea). Densitometric analysis was performed using Image J software (developed at US National Institutes of Health and available at http://rsb.info.nih.gov/ij/).

\section{Immunohistochemistry}

The animals were anesthetized with urethane $(1.2 \mathrm{~g} / \mathrm{kg}$, i.p.) and perfused transcardially with phosphate buffered saline (PBS) followed by $4 \%$ paraformaldehyde in $0.1 \mathrm{M}$ phosphate buffer (PB, $\mathrm{pH}$ 7.4). Brains were removed from the skull, post-fixed overnight in the same fixative, and then immersed in $30 \%$ sucrose in $0.1 \mathrm{M}$ PBS for 4 days at $4^{\circ} \mathrm{C}$. Brains were sectioned horizontally at $30 \mu \mathrm{m}$ on a freezing microtome (CM1850, Leica Microsystems; Nussloch $\mathrm{GmbH}$, Nussloch, Germany). Tissues were attached to silanecoated glass slides and stored at $-20^{\circ} \mathrm{C}$ until further processing. Slides containing DRN neurons were selected and rinsed with PBS (0.01 M, pH 7.4). Slides were subsequently blocked with CASblock Histochemical Reagent (008120; Life Technologies, Seoul, Korea) for $1 \mathrm{~h}$ at room temperature $\left(20^{\circ} \mathrm{C}-25^{\circ} \mathrm{C}\right)$ and incubated with primary antibodies (anti-THP2 for tryptophan hydroxylase 2; anti-MEL-1A/B-R for melatonin receptor 1 \& 2) overnight. Slides were then incubated for $2 \mathrm{~h}$ with anti-rabbit IgG (A21206, Alexa Fluor $488 \mathrm{H}+\mathrm{L}$; Invitrogen, Rockford, IL, USA) or antimouse IgG (A11032, Alexa Fluor $594 \mathrm{H}+\mathrm{L}$; Invitrogen) and rinsed with PBS containing $0.2 \%$ Triton X-100. All antibodies were diluted in CAS-block reagent. Slides were mounted with VECTASHIELD Antifade Mounting Medium with 4,6-diamidino2-phenylindole (DAPI, H-1200; Vector, Burlingame, CA, USA) and cover-slipped. Images were acquired using a fluorescent microscope (EVOS FL Imaging System; Life Technologies).

\section{Drugs and chemicals}

Melatonin and urethane were purchased from Sigma-Aldrich Korea. The following reagents and chemicals were used in this study: anti-OSR1, anti-WNK1, anti-WNK3 (A301-579A, A301515A, and A301-877A, respectively; Bethyl Laboratories Inc., Montgomery, TX, USA), anti-WNK2, anti-WNK4 (25910002 and NB600-284, respectively; Novus Biologicals, Littleton, CO, USA), anti-TPH2, anti-SPAK (ab111828 and ab79045, respectively; Abcam, Cambridge, UK), anti-KCC2, anti-NKCC1, anti-phosphoSPAK/OSR1 (07-432, AB3660P, and 07-2273, respectively; Millipore, Darmstadt, Germany), anti-nNOS (4236; Cell Signaling Technology, Danvers, MA, USA), anti-MEL-1A/B-R, anti- $\beta$-actin (sc-398788 and sc-47778, respectively; Santa Cruz Biotechnology, Dallas, TX, USA), and anti-HRP-conjugated secondary antibodies (SAB-100 and SAB-300; Enzo Life Science, Seoul, Korea).

\section{Data analysis}

All experiments were carried out with at least 10 sample preparations. All the data obtained from densitometry analysis were exported as table formatted with Microsoft Excel (Microsoft Corp., Seattle, WA, USA) formatted tables. All the graphs were drawn using Excel. The data were statistically analyzed using Excel macro. Unpaired Student's t-test was used to compare the differences between control and melatonin-treated groups (0.1, 1 , and $10 \mu \mathrm{M})$. Data are presented as the mean \pm standard error of the mean. $\mathrm{p}<0.05$ was considered statistically significant.

\section{RESULTS}

\section{Location of melatonin receptors and tryptophan hydroxylase 2 (TPH2) in the DRN}

We first examined the distribution of melatonin receptors in rat DRN serotonergic neurons. Double-staining immunohistochemistry was performed to elucidate the localization of melatonin receptors in TPH2-immunopositive serotonergic neurons. Melatonin receptor-positive immunoreactivity was observed in almost all TPH2-positive neurons (Fig. 1), suggesting that DRN serotoninergic neurons contain melatonin receptors (MT1 and/or MT2).

\section{Effect of melatonin on nNOS expression in DRN neurons}

To elucidate the effect of melatonin on NO-regulated WNKSPAK/OSR1-CCC signaling, the change in nNOS expression after treatment of DRN slices with melatonin was measured. Treatment with melatonin at concentrations higher than $\mathrm{nM}$ and/ or for incubation times longer than $4-5 \mathrm{~h}$ can cause nuclear DNA activation [39]. To examine the effect of melatonin on the expression of nNOS protein levels without causing any additional DNA or RNA damage, DRN slices were treated with $0.1,1$, or $10 \mu \mathrm{M}$ of melatonin for $4 \mathrm{~h}$. Treatment with $10 \mu \mathrm{M}$ of melatonin for $4 \mathrm{~h}$ decreased nNOS expression compared with that of control $(\mathrm{p}=$ 0.029 , Student's t-test, $\mathrm{n}=12$, Fig. 2).

\section{Effect of melatonin on the expression of WNKs and SPAK/OSR1 in DRN neurons}

After DRN slices were treated with $0.1,1$, or $10 \mu \mathrm{M}$ of melatonin for $4 \mathrm{~h}$, the change in protein expression of WNKs was measured. There were no significant changes in the expression of WNK1, 2, or 3 (Student's t-test, $\mathrm{n}=14-15$, Fig. 3B-D). However, treatment DRN slices with 1 and $10 \mu \mathrm{M}$ of melatonin for $4 \mathrm{~h}$ significantly increased WNK4 expression ( $p=0.035$, Student's $t$-test, $n=13$, Fig. 3E). 

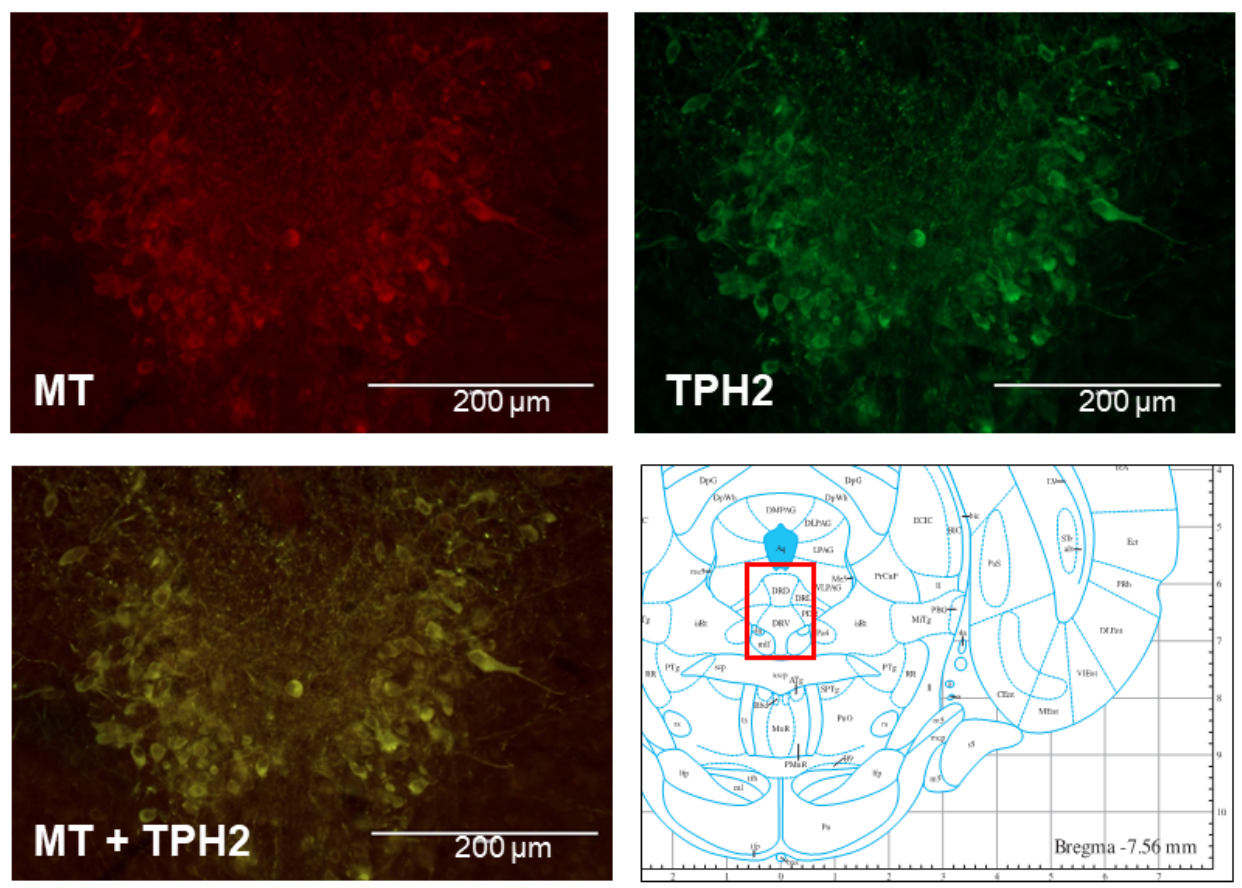

Fig. 1. Distribution of melatonin receptors (MT) and tryptophan hydroxylase 2 (TPH2)-containing serotonergic neurons in the dorsal raphe nucleus (DRN). Fluorescent images of melatonin receptors (including both MT1 and MT2, red) and TPH2-containing neurons (TPH, green). The bottom right images was taken from the rat brain atlas. The red square indicates the location of DRN. Scale bars: $200 \mu \mathrm{m}$.

A

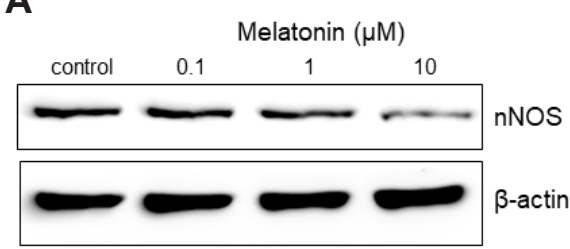

B

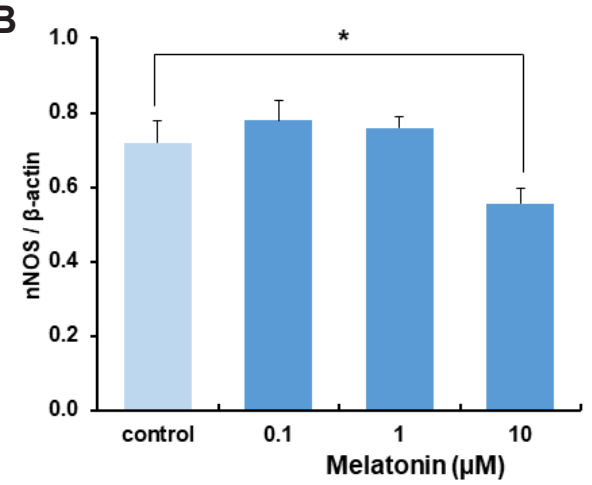

Fig. 2. Effect of melatonin on neuronal nitric oxide synthase (nNOS) expression in dorsal raphe nucleus (DRN) neurons. (A) Western blots showing nNOS expression in DRN serotonergic neurons following melatonin treatment $(0.1,1$, and $10 \mu \mathrm{M})$ for $4 \mathrm{~h}$. (B) Averaged optical density of nNOS expressions after $4 \mathrm{~h}$ melatonin incubation, expressed relative to control. Each column represents the mean and SEM of data from 12 experiments. ${ }^{*} p<0.05$ compared with control.
After treatment of DRN slices with $0.1,1$, or $10 \mu \mathrm{M}$ of melatonin, changes in SPAK/OSR1 protein expression were measured by Western blot. The expression of OSR1 and its phosphorylated form was significantly altered with $10-\mu \mathrm{M}$ melatonin treatment for $4 \mathrm{~h}$. Melatonin significantly decreased the expression of OSR 1 relative to that of the control $(\mathrm{p}=0.002$, Student's t-test, $\mathrm{n}=14$, Fig. 4C) and increased the expression of phosphorylated OSR1 compared to that of the control $(\mathrm{p}=0.016$, Student's $\mathrm{t}$-test, $\mathrm{n}=$ 14, Fig. 4E). The expression of phosphorylated and total form of SPAK was not altered with melatonin treatment (Student's t-test, $\mathrm{n}=15$, Fig. 4B, D).

\section{Effect of melatonin on NKCC1 and KCC2 expression in DRN neurons}

WNK-SPAK/OSR1 kinase regulates CCCs via the transducer SPAK/OSR1 kinases $[17,40]$. The WNK signaling pathway activates CCCs depending on phosphorylation $[17,41,42]$. We examined the effect of melatonin on NKCC1 and KCC2 expressions in
DRN neurons. NKCC1 expression was increased by the treatment of DRN slices with 1 and $10 \mu \mathrm{M}$ of melatonin for $4 \mathrm{~h}(\mathrm{p}=0.027$ and $p=0.000$, respectively; Student's t-test, $n=10$, Fig. $5 B$ ). There was no significant change in KCC2 expression with $0.1,1$, or 10 $\mu \mathrm{M}$ of melatonin treatment (Student's t-test, $\mathrm{n}=12$, Fig. 5 C).

\section{Effect of melatonin on tryptophan-hydroxylase-2 expression in DRN neurons}

Increased expression of $\mathrm{NKCC} 1$ with melatonin treatment may increase intracellular $\mathrm{Cl}^{-}$concentration and reduce GABAergic synaptic inhibition of DRN neurons, resulting in a net increase in the activity of DRN serotonergic neurons. Based on this possibility, we examined the effect of melatonin on TPH2 expression as an indicator of neuronal activity in DRN serotonergic neurons. Treatment of DRN slices with $10 \mu \mathrm{M}$ melatonin for $4 \mathrm{~h}$ significantly increased TPH2 expression ( $\mathrm{p}=0.049$, Student's t-test, $\mathrm{n}=$ 15; Fig. 6). 


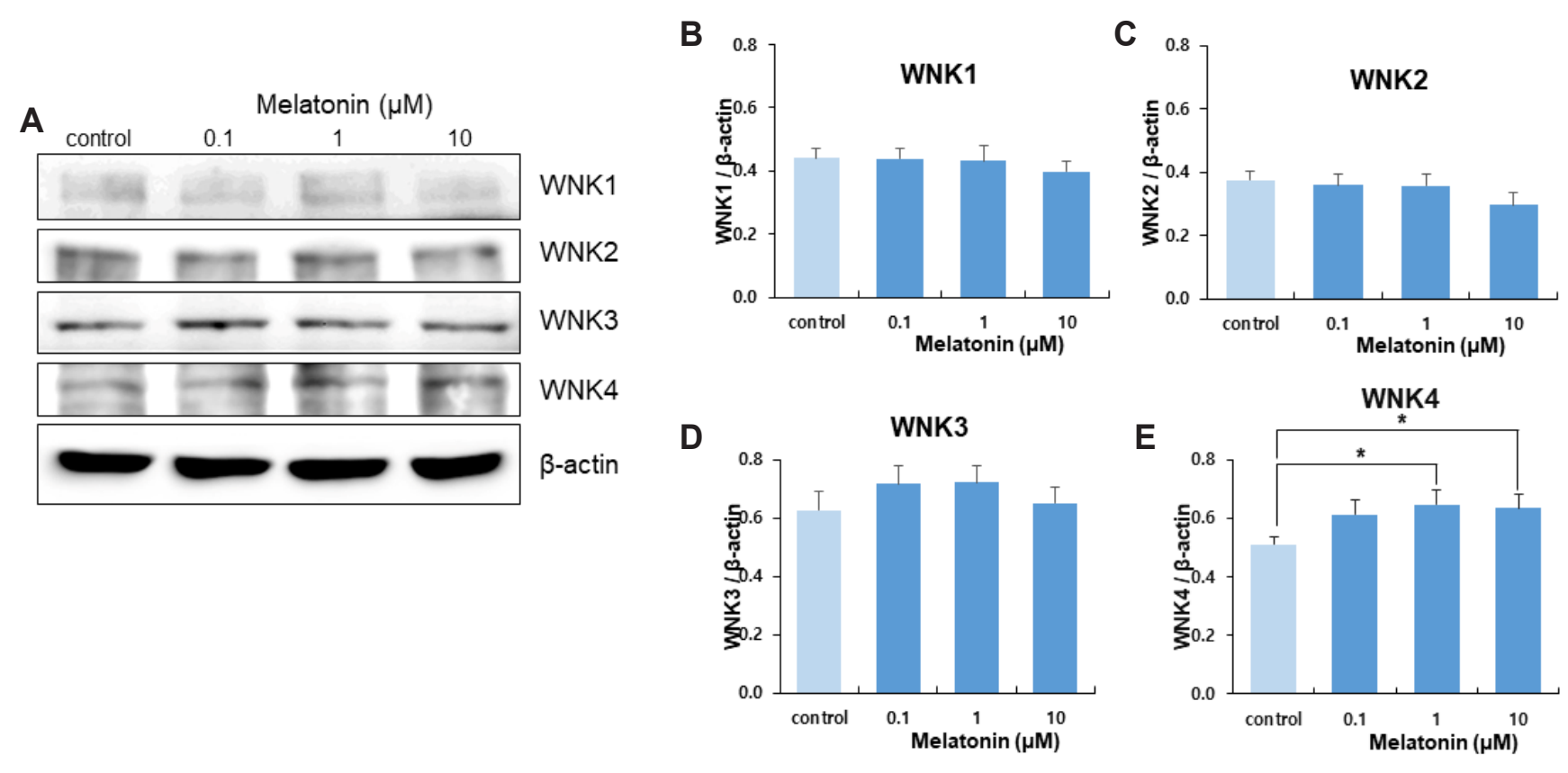

Fig. 3. Effect of melatonin on expression of with-no-lysine kinases (WNKs) in dorsal raphe nucleus (DRN) neurons. (A) Western blots showing changes in WNK1, 2, 3, and 4 expression in DRN slices after melatonin treatment $(0.1,1$, and $10 \mu \mathrm{M})$ for $4 \mathrm{~h}$. (B-E) Averaged optical density of WNK $1-4$ expressions after $4 \mathrm{~h}$ melatonin incubation, expressed relative to control. Each column represents the mean and SEM of data from 13-15 experiments. * $p<0.05$ compared with control.

A

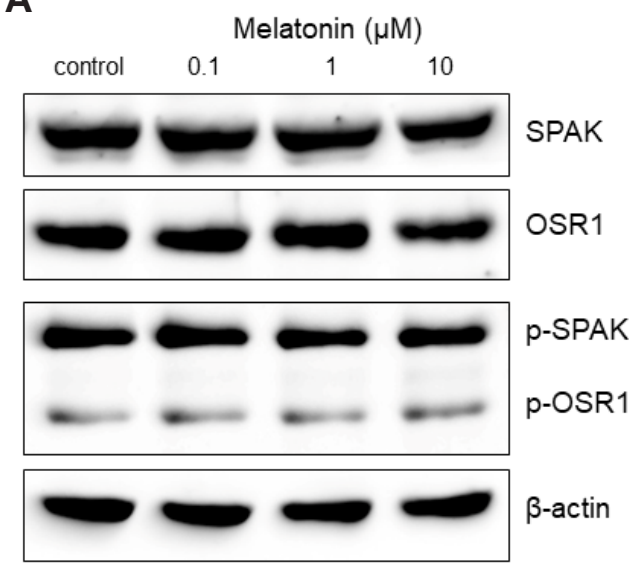

B

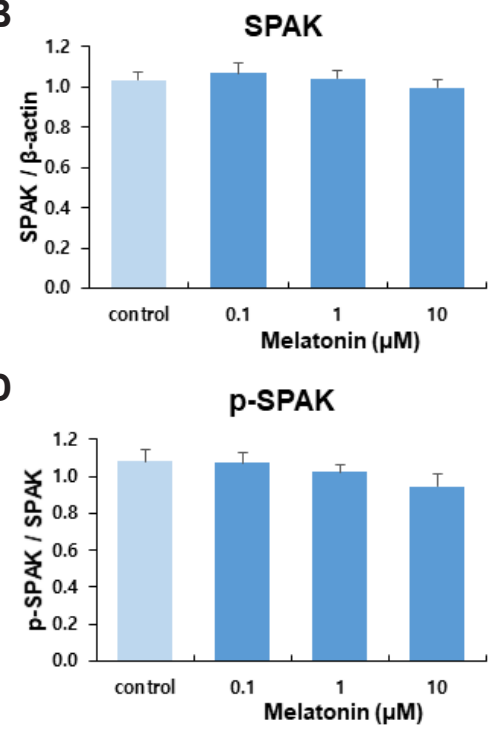

C

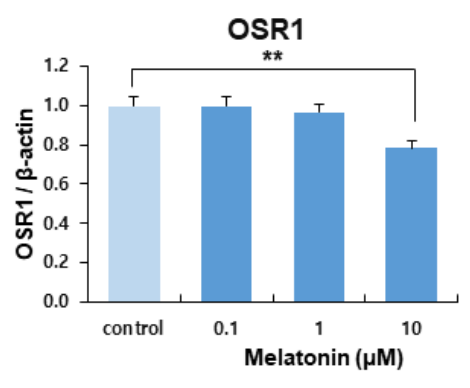

E

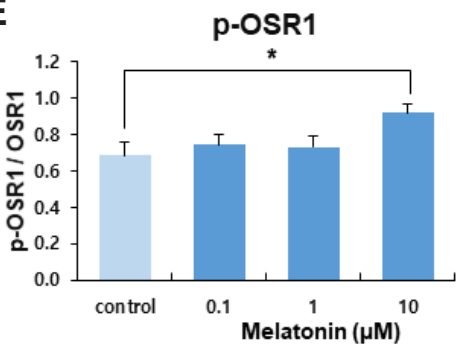

Fig. 4. Effect of melatonin on Ste20-related proline alanine rich kinase (SPAK)/oxidative stress response kinase 1 (OSR1) expression in dorsal raphe nucleus (DRN) neurons. (A) Western blots showing phospho- and total SPAK and OSR1 expression in DRN slices after melatonin treatment (0.1, 1, and $10 \mu \mathrm{M}$ ) for $4 \mathrm{~h}$. (B-E) Averaged optical density of phospho- and total-forms of SPAK/OSR1 expressions after $4 \mathrm{~h}$ melatonin incubation, expressed relative to control. Each column represents the mean and SEM of data from $14-15$ experiments. ${ }^{* *} p<0.01,{ }^{*} p<0.05$ compared with control.

\section{DISCUSSION}

The present study investigated the effect of melatonin on NOregulated WNK-SPAK/OSR1-CCC signaling in wake-inducing DRN neurons to elucidate the mechanism underlying melatonin's wake-promoting action in rats. Treatment of DRN slices with melatonin decreased nNOS expression and increased WNK4 expression without altering the expressions of WNK1, 2, or 3 . Melatonin increased OSR1 phosphorylation and NKCC1 expression without altering KCC2 expression, and increased TPH2 expression. The results suggest that melatonin promotes nighttime wakefulness through the modulation of NO-regulated WNK- 
A

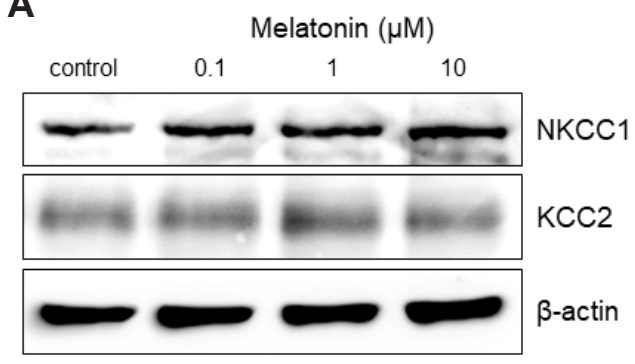

B
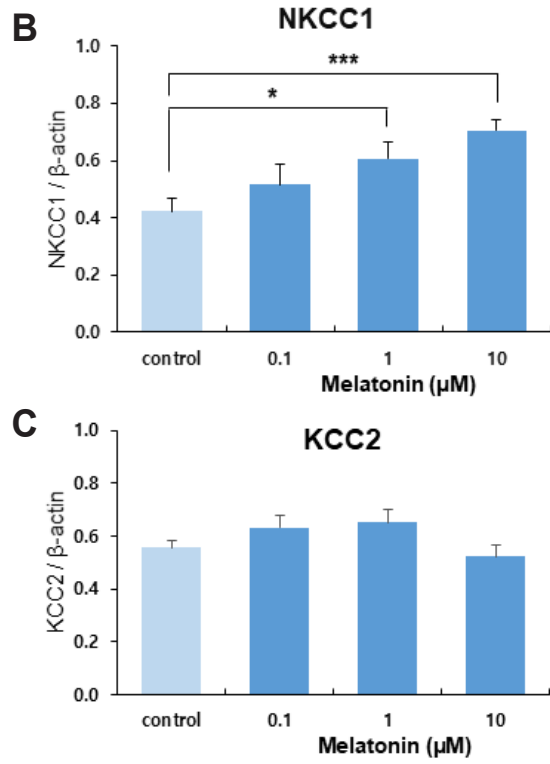

B

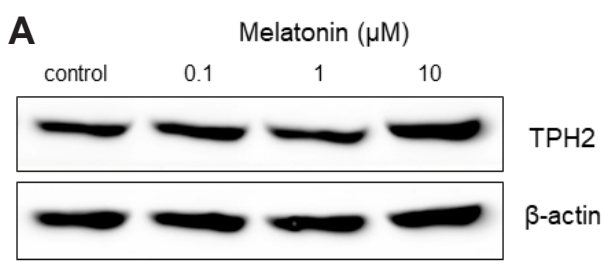

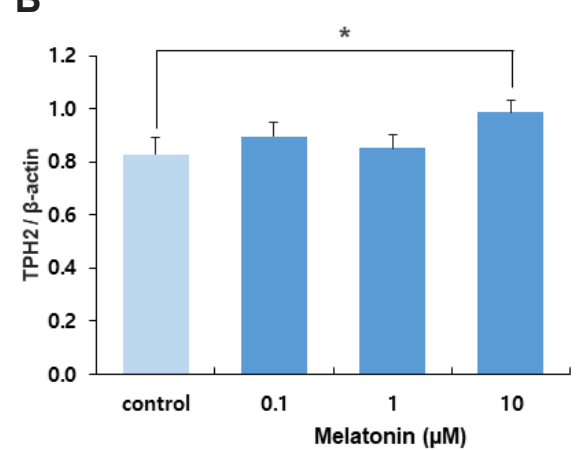

Fig. 5. Effect of melatonin on the expression of sodium-potassiumchloride co-transporter 1 (NKCC1) and potassium-chloride co-transporter 2 (KCC2) in dorsal raphe nucleus (DRN) neurons. (A) Western blots showing NKCC1 and KCC2 expression in DRN slices with melatonin treatment (0.1, 1 , and $10 \mu \mathrm{M})$ for $4 \mathrm{~h}$. Averaged optical density of NKCC1 (B) and KCC2 (C) expression after $4 \mathrm{~h}$ melatonin incubation, expressed relative to each control level. Each column represents the mean and SEM of data from 10-12 experiments. *** $p<0.001,{ }^{*} p<0.05$ compared with control.

Fig. 6. Effect of melatonin on tryptophan hydroxylase 2 (TPH2) expression in dorsal raphe nucleus (DRN) neurons. (A) Western blots showing $\mathrm{TPH} 2$ expression in DRN neurons with melatonin treatment $(0.1,1$, and $10 \mu \mathrm{M})$ for 4 h. (B) Averaged optical density of TPH2 expression, at $4 \mathrm{~h}$ melatonin incubation, expressed relative to control level of $\mathrm{TPH} 2$. Each column represents the mean and SEM of data from 15 experiments. ${ }^{*} p<0.05$ compared with control.
SPAK/OSR1-NKCC1 signaling in rat DRN serotonergic neurons.

In our recent report [24], we demonstrated that treatment of DRN neurons with a NO donor (sodium nitroprusside) for $4 \mathrm{~h}$ increases GABAergic synaptic inhibition and the expressions of WNK1, WNK2, WNK3, and KCC2, and decreases SPAK phosphorylation. In addition, experiments performed during the sleep-wake cycle showed that the expression of nNOS increased during daytime and decreased during nighttime. In this context, our results suggest that GABAergic synaptic inhibition of DRN serotonergic neurons changes daily during the sleep-wake cycle, which might be regulated by corresponding daily changes in nNOS-derived NO and WNK-SPAK/OSR1-KCC2 signaling in rats. However, our findings do not elucidate the mechanism that initiates the daily change in nNOS expression in rat DRN serotonergic neurons during the sleep-wake cycle.

In the present study, immunohistochemistry showed that melatonin receptor-positive immunoreactivity was observed in almost all TPH2-positive DRN serotonergic neurons (Fig. 1). Although the melatonin antibody used in this study was not selective to either the MT1 or MT2 receptor, our result indicates that melatonin can modulate the wake-inducing action of DRN serotonergic neurons.

Melatonin release peaks at midnight and dawn, and falls during the day [26]. High levels of melatonin release during the night induce sleep in humans [29] by promoting adenosine signaling, which is involved in circadian and homeostatic control of sleep in zebrafish [25]. On the other hand, melatonin reduced REM and SWS sleep and increased wakefulness in rats [30]. Given that melatonin release changes diurnally and promotes wakefulness in rats, we herein hypothesized that the high level of melatonin during the night decreases $\mathrm{nNOS}$ expression in rat DRN serotonergic neurons and that low melatonin levels during the day induces the reversed process. As expected, treatment of DRN slices with melatonin for $4 \mathrm{~h}$ decreased nNOS expression in rat DRN neurons (Fig. 2). This result is consistent with a report showing that melatonin treatment for a few hours inhibits nNOS [43].

As demonstrated in our recent report [24], a change of nNOS expression with melatonin can modulate NO-regulated WNKSPAK/OSR1-CCC signaling in rat DRN serotonergic neurons. In this study, treatment of DRN slices with melatonin for $4 \mathrm{~h}$ increased WNK4 expression but did not alter the expressions of WNK1, 2, or 3 (Fig. 3). WNK kinases phosphorylate downstream 


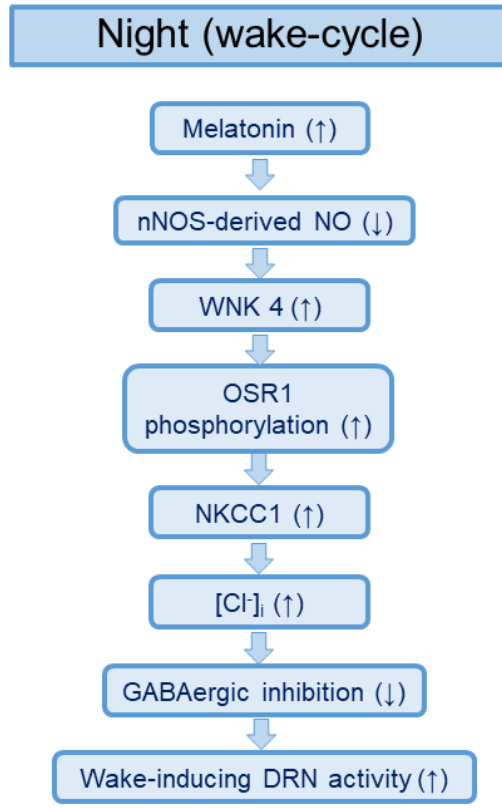

\section{Day (sleep-cycle)}

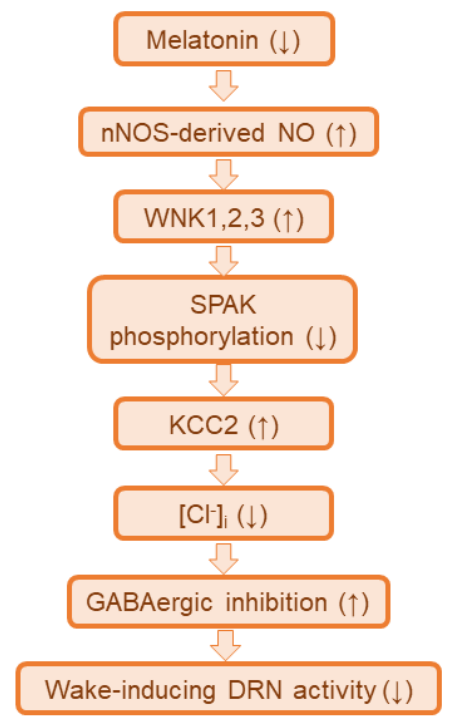

Fig. 7. Schematic diagram showing a possible suggestion of the signaling pathways by which melatonin modulates NO-regulated WNKSPAK/OSR1-CCC signaling during the sleep-wake cycle in rat DRN serotonergic neurons. NO, nitric oxide; WNK, with-no-lysine kinase; SPAK, Ste20-related proline alanine rich kinase; OSR1, oxidative stress response kinase 1; CCC, cation-chloride co-transporters; DRN, dorsal raphe nucleus; nNOS, neuronal nitric oxide synthase; NKCC1, sodium-potassium-chloride co-transporter 1; KCC2, potassium-chloride co-transporter 2.

SPAK/OSR1 kinases, which modulate NKCC1 or KCC2 activity [15-19]. Treatment of DRN slices with melatonin for $4 \mathrm{~h}$ increased the phosphorylation of OSR1 without altering SPAK phosphorylation (Fig. 4); this treatment also increased NKCC1 expression without altering KCC2 (Fig. 5). Our data indicate that WNK4 phosphorylates OSR1, which then upregulates NKCC1 in rat DRN neurons. The results are consistent with previous reports that WNK4 phosphorylates and activates NKCC1 activity in Xenopus laevis oocytes [40].

WNK kinases phosphorylate KCC and decrease its activity, while WNKs phosphorylate NKCC and increase its activity $[17,44]$. NKCC1 and KCC2 regulate plasma membrane proteins that modulate postsynaptic actions $[15,45,46]$ and regulate intracellular $\mathrm{Cl}^{-}$concentration, which modulates the inhibitory effect of GABAergic synaptic inputs [12-14]. The DRN is the origin of the central 5-HT system [9,47], and its neuronal activity is modulated by GABAergic synaptic inputs [11]. In the present study, the melatonin-induced upregulation of NKCC1 may increase intracellular $\mathrm{Cl}^{-}$concentration and attenuate the inhibitory GABAergic synaptic action in rat DRN serotonergic neurons. Decreased GABAergic inhibition may augment 5-HT expression in DRN serotonergic neurons and enhance the wake-inducing activity of DRN serotonergic neurons during the night. We showed that the treatment of DRN slices with melatonin for $4 \mathrm{~h}$ increased TPH2 expression in rat DRN neurons (Fig. 6), which may suggest that melatonin may activate the wake-promoting action of rat DRN serotonergic neurons through its activation of WNK-SPK/OSR1NKCC1 signaling.

The results of the present study establish a possible role of melatonin as an initiating regulator of NO-regulated WNKSPAK/OSR1-CCC signaling in rat DRN serotonergic neurons. Meanwhile, our recent study demonstrated that nNOS expression in rat DRN neurons increased during the day and decreased during the night and that treatment of DRN slices with an NO donor increased GABAergic synaptic inhibition and the expressions of nNOS, KCC2, WNK 1, WNK 2, and WNK 3, and decreased SPAK phosphorylation [24]. Taken together, these findings have contributed to a working model in which high melatonin decreases nNOS expression and increases WNK4 kinase expression during the night, thereby increasing NKCC1 levels through OSR1 phosphorylation. The increased expression of NKCC1 might be suggested to attenuate the inhibitory GABAergic action, which enhances the wake-promoting action of DRN serotonergic neurons (Fig. 7). On the other hand, suppressed melatonin levels during the day might be suggested to increase nNOS expression, which activates the WNK-SPAK/OSR1-KCC2 signaling pathway. Increased KCC2 expression might be suggested to enhance the inhibitory GABAergic action and lead to the inhibition of the wake-promoting action of DRN serotonergic neurons (Fig. 7). In conclusion, the present study suggests that melatonin exerts its wake-promoting actions through the activation of NO-regulated WNK-SPAK/OSR1-NKCC1 signaling pathway in rat DRN serotonergic neurons, and that melatonin acts as an initiating regulator of nNOS expression in the NO-regulated WNK-SPAK/OSR1CCC signaling pathway during the sleep-wake cycle.

Future studies are required to clearly elucidate the following: which melatonin receptor subtype is involved in the effects of melatonin on the NO-related WNK-SPAK/OSR1-NKCC1 signal- 
ing in rat $\mathrm{DRN}$ serotonergic neurons, the molecular mechanisms underlying the effects of melatonin on the expressions of nNOS and WNK4, the electrical change in GABAergic synaptic inhibition of DRN serotonergic neurons, and changes in SPAK/OSR1phosphorylation sites on NKCC1. In addition, although we used both sexes of rats in all experiments of the present study, further studies will be helpful to elucidate a possibility of sex differences in the results of this study.

\section{ACKNOWLEDGEMENTS}

This work was supported by a National Research Foundation of Korea (NRF) grant funded by the Korean government (MSIP) (nos. 2011-0030072 and 2011-0011939).

\section{CONFLICTS OF INTEREST}

The authors declare no conflicts of interest.

\section{REFERENCES}

1. Xie L, Kang H, Xu Q, Chen MJ, Liao Y, Thiyagarajan M, O'Donnell J, Christensen DJ, Nicholson C, Iliff JJ, Takano T, Deane R, Nedergaard M. Sleep drives metabolite clearance from the adult brain. Science. 2013;342:373-377.

2. Goldstein AN, Walker MP. The role of sleep in emotional brain function. Annu Rev Clin Psychol. 2014;10:679-708.

3. Vyazovskiy VV, Harris KD. Sleep and the single neuron: the role of global slow oscillations in individual cell rest. Nat Rev Neurosci. 2013;14:443-451.

4. Fuller PM, Gooley JJ, Saper CB. Neurobiology of the sleep-wake cycle: sleep architecture, circadian regulation, and regulatory feedback. J Biol Rhythms. 2006;21:482-493.

5. Gallopin T, Fort P, Eggermann E, Cauli B, Luppi PH, Rossier J, Audinat E, Mühlethaler M, Serafin M. Identification of sleep-promoting neurons in vitro. Nature. 2000;404:992-995.

6. Lu J, Bjorkum AA, Xu M, Gaus SE, Shiromani PJ, Saper CB. Selective activation of the extended ventrolateral preoptic nucleus during rapid eye movement sleep. J Neurosci. 2002;22:4568-4576.

7. Brown RE, Basheer R, McKenna JT, Strecker RE, McCarley RW. Control of sleep and wakefulness. Physiol Rev. 2012;92:1087-1187.

8. Sakai K. Sleep-waking discharge profiles of dorsal raphe nucleus neurons in mice. Neuroscience. 2011;197:200-224.

9. Monti JM. The role of dorsal raphe nucleus serotonergic and nonserotonergic neurons, and of their receptors, in regulating waking and rapid eye movement (REM) sleep. Sleep Med Rev. 2010;14:319327.

10. Monti JM, Jantos H. The roles of dopamine and serotonin, and of their receptors, in regulating sleep and waking. Prog Brain Res. 2008;172:625-646.

11. Gervasoni D, Peyron C, Rampon C, Barbagli B, Chouvet G, Urbain N, Fort P, Luppi PH. Role and origin of the GABAergic innervation of dorsal raphe serotonergic neurons. J Neurosci. 2000;20:42174225.

12. Payne JA, Rivera C, Voipio J, Kaila K. Cation-chloride co-transporters in neuronal communication, development and trauma. Trends Neurosci. 2003;26:199-206.

13. Kaila K. Ionic basis of GABAA receptor channel function in the nervous system. Prog Neurobiol. 1994;42:489-537.

14. Kaila K, Price TJ, Payne JA, Puskarjov M, Voipio J. Cation-chloride cotransporters in neuronal development, plasticity and disease. Nat Rev Neurosci. 2014;15:637-654.

15. Blaesse P, Airaksinen MS, Rivera C, Kaila K. Cation-chloride cotransporters and neuronal function. Neuron. 2009;61:820-838.

16. Alessi DR, Zhang J, Khanna A, Hochdörfer T, Shang Y, Kahle KT. The WNK-SPAK/OSR1 pathway: master regulator of cation-chloride cotransporters. Sci Signal. 2014;7:re3.

17. Kahle KT, Rinehart J, Lifton RP. Phosphoregulation of the Na-K$2 \mathrm{Cl}$ and $\mathrm{K}-\mathrm{Cl}$ cotransporters by the WNK kinases. Biochim Biophys Acta. 2010;1802:1150-1158.

18. Piechotta K, Lu J, Delpire E. Cation chloride cotransporters interact with the stress-related kinases Ste20-related proline-alanine-rich kinase (SPAK) and oxidative stress response 1 (OSR1). J Biol Chem. 2002;277:50812-50819.

19. Thastrup JO, Rafiqi FH, Vitari AC, Pozo-Guisado E, Deak M, Mehellou Y, Alessi DR. SPAK/OSR1 regulate NKCC1 and WNK activity: analysis of WNK isoform interactions and activation by T-loop trans-autophosphorylation. Biochem J. 2012;441:325-337.

20. Ayers NA, Kapás L, Krueger JM. Circadian variation of nitric oxide synthase activity and cytosolic protein levels in rat brain. Brain Res. 1996;707:127-130.

21. Chen L, Majde JA, Krueger JM. Spontaneous sleep in mice with targeted disruptions of neuronal or inducible nitric oxide synthase genes. Brain Res. 2003;973:214-222.

22. Kalinchuk AV, Stenberg D, Rosenberg PA, Porkka-Heiskanen T. Inducible and neuronal nitric oxide synthases (NOS) have complementary roles in recovery sleep induction. Eur J Neurosci. 2006;24:1443-1456.

23. Cespuglio R, Amrouni D, Meiller A, Buguet A, Gautier-Sauvigné $\mathrm{S}$. Nitric oxide in the regulation of the sleep-wake states. Sleep Med Rev. 2012;16:265-279.

24. Kim MJ, Yang HJ, Kim Y, Kang I, Kim SS, Cho YW. Role of nitric oxide and WNK-SPAK/OSR1-KCC2 signaling in daily changes in GABAergic inhibition in the rat dorsal raphe neurons. Neuropharmacology. 2018;135:355-367.

25. Gandhi AV, Mosser EA, Oikonomou G, Prober DA. Melatonin is required for the circadian regulation of sleep. Neuron. 2015;85:11931199.

26. Tan DX, Manchester LC, Esteban-Zubero E, Zhou Z, Reiter RJ. Melatonin as a potent and inducible endogenous antioxidant: synthesis and metabolism. Molecules. 2015;20:18886-18906.

27. Szabadi E. Drugs for sleep disorders: mechanisms and therapeutic prospects. Br J Clin Pharmacol. 2006;61:761-766.

28. Schwartz MD, Wotus C, Liu T, Friesen WO, Borjigin J, Oda GA, de la Iglesia HO. Dissociation of circadian and light inhibition of melatonin release through forced desynchronization in the rat. Proc Natl Acad Sci U S A. 2009;106:17540-17545.

29. Cajochen C, Kräuchi K, Wirz-Justice A. Role of melatonin in the regulation of human circadian rhythms and sleep. J Neuroendocri- 
nol. 2003;15:432-437.

30. Descamps A, Rousset C, Millan MJ, Spedding M, Delagrange P, Cespuglio R. Influence of the novel antidepressant and melatonin agonist/serotonin $2 \mathrm{C}$ receptor antagonist, agomelatine, on the rat sleep-wake cycle architecture. Psychopharmacology (Berl). 2009;205:93-106.

31. Pevet P, Challet E. Melatonin: both master clock output and internal time-giver in the circadian clocks network. J Physiol Paris. 2011;105:170-182.

32. Vriend J, Reiter RJ. Melatonin feedback on clock genes: a theory involving the proteasome. J Pineal Res. 2015;58:1-11.

33. von Gall C, Stehle JH, Weaver DR. Mammalian melatonin receptors: molecular biology and signal transduction. Cell Tissue Res. 2002;309:151-162.

34. Weaver DR, Reppert SM. The Mella melatonin receptor gene is expressed in human suprachiasmatic nuclei. Neuroreport. 1996;8:109112.

35. Kim JS, Kim WB, Kim YB, Lee Y, Kim YS, Shen FY, Lee SW, Park D, Choi HJ, Hur J, Park JJ, Han HC, Colwell CS, Cho YW, Kim YI. Chronic hyperosmotic stress converts GABAergic inhibition into excitation in vasopressin and oxytocin neurons in the rat. J Neurosci. 2011;31:13312-13322.

36. Ruusuvirta T, Lipponen A, Pellinen EK, Penttonen M, Astikainen P. Auditory cortical and hippocampal local-field potentials to frequency deviant tones in urethane-anesthetized rats: an unexpected role of the sound frequencies themselves. Int J Psychophysiol. 2015;96:134-140.

37. Paxinos G, Watson C. The rat brain in stereotaxic coordinates. Cambridge: Academic Press; 2007.

38. Hur J, Lee P, Kim MJ, Cho YW. Regulatory effect of 25-hydroxyvitamin D3 on nitric oxide production in activated microglia. Korean J Physiol Pharmacol. 2014;18:397-402.

39. Arese M, Magnifico MC, Mastronicola D, Altieri F, Grillo C, Blanck
TJ, Sarti P. Nanomolar melatonin enhances nNOS expression and controls HaCaT-cells bioenergetics. IUBMB Life. 2012;64:251-258.

40. Gagnon KB, England R, Delpire E. Volume sensitivity of cationCl- cotransporters is modulated by the interaction of two kinases: Ste20-related proline-alanine-rich kinase and WNK4. Am J Physiol Cell Physiol. 2006;290:C134-C142.

41. de Los Heros P, Kahle KT, Rinehart J, Bobadilla NA, Vázquez N, San Cristobal P, Mount DB, Lifton RP, Hebert SC, Gamba G. WNK3 bypasses the tonicity requirement for $\mathrm{K}-\mathrm{Cl}$ cotransporter activation via a phosphatase-dependent pathway. Proc Natl Acad Sci U S A. 2006;103:1976-1981.

42. Rinehart J, Maksimova YD, Tanis JE, Stone KL, Hodson CA, Zhang J, Risinger M, Pan W, Wu D, Colangelo CM, Forbush B, Joiner CH, Gulcicek EE, Gallagher PG, Lifton RP. Sites of regulated phosphorylation that control K-Cl cotransporter activity. Cell. 2009;138:525536.

43. Sarti P, Magnifico MC, Altieri F, Mastronicola D, Arese M. New evidence for cross talk between melatonin and mitochondria mediated by a circadian-compatible interaction with nitric oxide. Int $J$ Mol Sci. 2013;14:11259-11276.

44. Kahle KT, Rinehart J, Ring A, Gimenez I, Gamba G, Hebert SC, Lifton RP. WNK protein kinases modulate cellular $\mathrm{Cl}$ - flux by altering the phosphorylation state of the $\mathrm{Na}-\mathrm{K}-\mathrm{Cl}$ and $\mathrm{K}-\mathrm{Cl}$ cotransporters. Physiology (Bethesda). 2006;21:326-335.

45. Kahle KT, Deeb TZ, Puskarjov M, Silayeva L, Liang B, Kaila K, Moss SJ. Modulation of neuronal activity by phosphorylation of the K-Cl cotransporter KCC2. Trends Neurosci. 2013;36:726-737.

46. Rivera C, Voipio J, Payne JA, Ruusuvuori E, Lahtinen H, Lamsa K, Pirvola U, Saarma M, Kaila K. The $\mathrm{K}^{+} / \mathrm{Cl}^{-}$co-transporter $\mathrm{KCC} 2$ renders GABA hyperpolarizing during neuronal maturation. Nature. 1999;397:251-255.

47. Monti JM. Serotonin control of sleep-wake behavior. Sleep Med Rev. 2011;15:269-281. 